\title{
Evidence-based Analysis of Psychological Intervention for Menopausal Syndrome
}

\author{
Xuemei $\mathrm{Yi}^{1}$ \\ Psychological Counseling and Instruction Center \\ Jiangxi University of Traditional Chinese Medicine \\ Nanchang, China
}

\author{
Ting Ding $2 *$ \\ Gynecology Department \\ Ruichang Hospital of Traditional Chinese Medicine \\ Ruichang, China
}

\begin{abstract}
The research aims to systematically evaluate the effect of psychological intervention on perimenopausal syndrome. We searched CNKI, VIP, WanFang Data and China Biomedical Literature Database to collect randomized controlled trials on psychological intervention combined with drugs for menopausal syndrome. After data extraction and quality evaluation using exclusion criteria, a total of $\mathbf{2 8}$ articles were included, with $\mathbf{2 2 6 4}$ patients. Meta-analysis showed (1) in symptoms: Kupperman scores with psychological intervention decreased more significant than drug treatment $[\mathrm{MD}=5.55,95 \% \mathrm{CI}(4.76,6.34), \mathrm{P}<0.00001]$; (2) in emotions: $\mathrm{SAS}[\mathrm{MD}=8.54,95 \% \mathrm{CI}(6.21,10.86), \mathrm{P}<0.0001]$ and SDS $[M D=12.20,95 \% \mathrm{CI}(8.33,16.08, \quad \mathrm{P}<0.00001]$ decreased more significant than drugs; (3) in the total efficiency: psychological intervention after total curative effect is obviously better than simple drug treatment $[O R=7.32,95 \% \mathrm{CI}(5.11,10.50), P<0.00001]$ (4) in hormone: it showed no differences in E2 and FSH levels whether before or after psychological intervention, while for $\mathbf{L H}$, the results stayed different. In conclusion, combined therapy of menopausal syndrome showed great effects on improving symptoms, efficacies, and negative emotions, but not for hormones.
\end{abstract}

Keywords—psychological intervention; perimenopause; systematic evaluation; randomized controlled trial

\section{INTRODUCTION}

"Perimenopausal Syndrome" refers to groups of symptoms characterized by dysfunction of autonomic nervous system and neuropsychological symptoms caused by decline of hormone secretion, imbalance of hypothalamus-pituitaryovary. About $2 / 3$ of women would have perimenopausal syndrome ${ }^{[1]}$, mostly occurring between 40 and 60 . Sudden drop of estrogen in menopausal women causes a series of physiological changes, as insomnia, depression, anxiety, etc, which seriously affect their physical, mental health and wellbeing of themselves. Women have entered menopause ahead of time moreover ${ }^{[2]}$. It's of great effect to early detection of climacteric syndrome, appropriate treatment and effective psychological intervention. Symptomatic treatment, hormone replacement therapy(e.g. nilestriol,etc) and antidepressant therapy(e.g.doxepin) are mostly used in drug therapy to relieve symptoms of patients. However, hormone therapy has many contraindications and side effects, and facts proved it cannot completely relieve or alleviate symptoms, more seriously, there are much potential risks as breast cancer, cardiovascular diseases, etc, if long-term HRT treatments ${ }^{[3]}$. In addition, risks of endometrial cancer, cervical cancer and

Supported by: National Innovation and Enterpriseship Training Program (201710412004) and Scientifac Researches Program of JXUTCM

(2016jzzdxk011).. hysteromyoma increased accordingly. In contrast, psychological intervention has advantages of wide adaptability and less side effects.

Many reports have reported the efficacy of psychological intervention in the treatment of menopausal syndrome, but few of them strictly and systematically evaluated, besides with great differences and unauthentic evidences, which thus brings certain difficulties to the evidence-based decisionmaking of clinical treatment.

\section{DATA AND METHODS}

\section{A. Inclusion criteria}

(1) Research Type: All randomized controlled trials (RCT) on psychological therapies for menopausal syndrome.

(2) Reaearch Subjects: Menopausal female patients, 40 and 60 years old, with various degrees of climacteric symptoms, accordance with diagnostic criteria of menopausal syndrome.

(3) Intervention Methods: Test group adopts psychological intervention combined with drugs, such hormone, doxepin and oryzanol); Control group treated with western medicine only.

(4) Result indicators: Each indicator adopted change value before and after treatment (pre-treatment Vs post-treatment score). Indicators included: (1)Symptom Checklist 90(SCL-90) anxiety factor; (2) SCL-90 depression factor; (3) Self-Rating Anxiety Scale(SAS); (4)Self-Rating Depression Scale(SDS); (5) Kupperman (climacteric symptoms); (6) Serum hormone level: blood estradiol (E2), follicle stimulating hormone (FSH) level and luteinizing hormone (LH); (7)Total efficiency rate= recovery rate+efficiency rate+effective rate, which recovery means all clinical symptoms disappear, efficiency means clinical symptoms obviously improved, and effectiveness means some clinical symptoms are improved. Inefficiency means clinical symptoms don't improve or worsen.

\section{B. Retrieval strategy}

Literatures were searched from CNKI, VIP, WanFang and China Biomedical Literature Databases, until to April 1, 2014. Search terms included "climacteric syndrome", "syndrome", "climacteric", "perimenopausal syndrome", "menopausal", "menopausal syndrome".

All the words were linked by OR. A total of 93,008 articles related were initially retrieved, and 38,973 remained 
after weight removal. What's more, 855 articles of psychological intervention were included after grouping and classification.

\section{Statistical Analysis}

RevMan 5.0.2 software was used, and measurement data are expressed by mean difference (MD) and 95\% CI, while calculated data are expressed by ratio (OR) and 95\%CI. Heterogeneity tests were carried out for each study, which fixed effect model was used to calculate the combined effect amount if no heterogeneity or small $\left(\mathrm{P}>0.1, \mathrm{I}^{2}<50 \%\right)$. However, if the heterogeneity is large ( $\left.\mathrm{P} \leq 0.1, \mathrm{I}^{2} \geq 50 \%\right)$, subgroup analysis should be carried out according to the factors causing heterogeneity (different treatment methods, different results evaluation time, etc.), so did analysed with sources and causes of heterogeneity.

\section{RESULTS}

\section{A. Literature search}

Our search of psychological intervention of premenopausal syndrome yielded 855 citations, and1 158 RCTs were screened out strictly according to the exclusion criteria, what's more, 96 RCTs were obtained for full-text review. After more clearly grouping analysis, the search strategy identified 34 citations. Of these, 28 were eligible for inclusion. Six studies were excluded for their incomplete datas. Flow diagram process and methodological quality assessment were showed in Fig. 1 \& Table I.

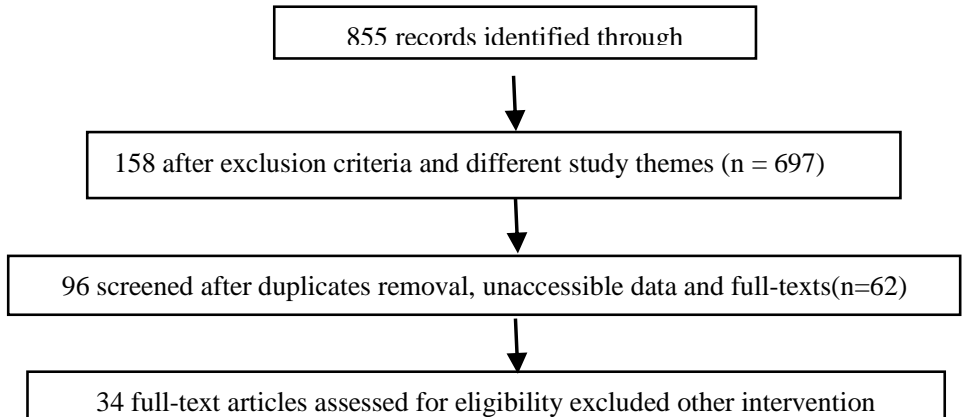

34 full-text articles assessed for eligibility excluded other intervention

6 full-text articles excluded for inconsistencies in data

28 studies included in Meta-

Fig. 1. Flow diagram of process for identification of studies

\section{B. Results of Meta-Analysis}

1) SCL-90 anxiety factor scores

Twelve articles ${ }^{[2,6-9,15,16,18,19,21,23,24]}, 895$ patients, compared the SCL-90 anxiety factor. It existed heterogeneity $\left(\mathrm{I}^{2}=99 \%\right)$, and subgroup analysis was carried out according to the measurement time (January, February, March and June), and statistically significant differences found exactly(MD=0.27,95\%CI $(0.04,0.49), \mathrm{P}=0.02)$.

\section{2) SCL-90 depression factor scores}

Twelve articles including 895 patients reported depression factor scores ${ }^{[2,6-9,15,16,18,19,21,23,24]}$. Heterogeneity $\left(\mathrm{I}^{2}=92 \%\right)$ was found and a randomed effects model was used to make analysis. It showed statistically significant differences $(\mathrm{MD}=0.28,95 \% \mathrm{CI}(0.15,0.41), \mathrm{P}<0.0001)$.

\section{3) Scores of SAS scale}

Five articles reported scores of SAS scale, 402 patients with heterogeneity $(\mathrm{I} 2=92 \%)^{[2,17,20,28,29]}$. Subgroup analysis was conducted to reveal significant differences in SAS scores $(\mathrm{MD}=8.54,95 \% \mathrm{CI}(6.21,10.86), \mathrm{p}<0.00001)$, showing combined psychological intervention was better than drug in improving anxiety of perimenopausal syndrome patients.

4) Scores of SDS scale

Six articles including 458 patients compared scores of SDS of two groups. It had heterogeneity $\left(\mathrm{I}^{2}=99 \%\right)$, and we randomed model was used ${ }^{[2,12,17,20,28,29]}$., with statistical difference $(\mathrm{MD}=8.54,95 \% \mathrm{CI}(6.21,10.86), \mathrm{P}<0.00001)$.

\section{5) Kupperman menopausal scores}

Three studies reported Kupperman scores, totaling 240 patients $^{[19,23,31]}$. There is no heterogeneity in the included study $(\mathrm{I} 2=0 \%)$, and results showed differences was statistically significant $\quad(\mathrm{MD}=5.55, \quad 95 \% \quad \mathrm{CI}(4.76$, $6.34), \mathrm{p}<0.00001)$.

6) Serum hormone levels

Two reports including 269 patients reported serum hormone levels ${ }^{[25,27]}$.It showed heterogeneity in E2 (I2=70\%), and meta-analysis results showed no statistically significant (MD=9.18, 95\% CI(-7.96,26.32), $\mathrm{P}=0.29)$. So did the heterogeneity in FSH and no statistically significant $(\mathrm{I} 2=98 \%), \mathrm{MD}=8.69,95 \% \mathrm{CI}(-$ 7.99,25.37), $\mathrm{P}=0.31)$. However, There was no heterogeneity in $\mathrm{LH}$ level($(\mathrm{I} 2=0 \%)$, and meta-analysis showed significant difference $(\mathrm{MD}=2.31,95 \%$ CI(1.17,3.45), $\mathrm{P}<0.0001)$.

\section{7) Total effective rate}

Twelve, including $\quad 1000 \quad$ patients $^{[5,8,10,11,13-}$ $16,22,25,26,30]$ reported the total effective rate of psychological therapy for menopausal syndrome,. No heterogeneity found $\left(\mathrm{I}^{2}=0 \%\right)$, but with statistically significant difference between groups $(\mathrm{OR}=7.32,95 \% \mathrm{CI}(5.11,10.50), \mathrm{p}<0.00001]$, which indicates the total effective rate of psychological intervention combined with drug therapy for menopausal syndrome patients was better than that of drug therapy alone. All the results showed in Table III.

\section{Funnel chart analysis}

We checked the total effective rates for all 28 citations using funnel chart analysis. Funnel plot was drawn with OR value as the abscissa and $\mathrm{SE}(\log \mathrm{OR})$ as the ordinate. The corresponding points were within the $95 \%$ confidence interval, but graph was not completely symmetrical distribution, indicating a small existence of publication bias(Figure.2). 


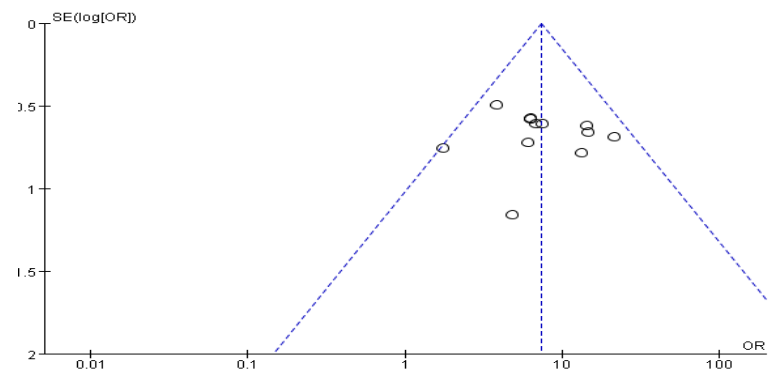

Fig. 2. Results of literature publication bias analysis

TABLE I

BASELINE PATIENT CHARACTERISTICS OF INCLUDED STUDIES

\begin{tabular}{|c|c|c|c|c|c|}
\hline \multirow[t]{2}{*}{ Study } & \multirow[t]{2}{*}{$\mathrm{n}(\mathrm{T} / \mathrm{C})$} & \multicolumn{2}{|c|}{ Treatment } & \multirow[t]{2}{*}{ Course } & \multirow[t]{2}{*}{ Result Index } \\
\hline & & $\mathrm{T}$ & $\mathrm{C}$ & & \\
\hline Mo et al 2014 & $50 / 50$ & Psychology combined medicines & Medicine & 2 months & (7) \\
\hline Deng et al 2004 & $50 / 50$ & Psychology combined medicines & Medicine & 1 months & (1)(2) \\
\hline Meng et al 2011 & $35 / 35$ & Psychology combined medicines & Medicine & 3 months & (1)(2) \\
\hline Zhang et al 2006 & $33 / 31$ & Psychology combined medicines & Medicine & 2 months & (1)(2)(7) \\
\hline Lin et al 2012 & $80 / 40$ & Psychology combined medicines & Medicine & 6 months & (1)(2) \\
\hline Shen et al 2002 & $40 / 40$ & Psychology combined medicines & Medicine & 2 months & (7) \\
\hline Zhang et al 2012 & $60 / 60$ & Psychology combined medicines & Hormone therapy & Not stated & (7) \\
\hline Li et al 2002 & $28 / 28$ & Psychology combined medicines & Hormone therapy & Not stated & (4) \\
\hline Li et al 2009 & $40 / 40$ & Psychology combined medicines & Medicine & Not stated & (7) \\
\hline Cui et al 2007 & $69 / 34$ & Psychology combined medicines & Medicine & 2 months & (7) \\
\hline Liu et al 2012 & $31 / 31$ & Psychology combined medicines & Fluoxetine & 2 months & (1)(2)(7) \\
\hline Liu et al 2003 & $32 / 31$ & Psychology combined medicines & Doxepin & 2 months & (1)(2)(7) \\
\hline Xu et al 2012 & $49 / 49$ & Psychology combined medicines & Progesterone Treatment & 2 months & (3)(4) \\
\hline Tian et al 2007 & $25 / 21$ & Psychology combined medicines & Oryzanol and doxepin & 2 months & (1)(2) \\
\hline Yan et al 2009 & $34 / 34$ & Psychology combined medicines & Medicine & Not stated & (1)(2)(3)(4) \\
\hline Hu et al 2007 & $30 / 30$ & Consult combined hormone therapy & Medicine & 1 months & (1)(2)(5) \\
\hline Zou et al 2013 & $54 / 49$ & Psychology combined medicines & Medicine & 1 months & (3)(4) \\
\hline Wang et al 2008 & $40 / 40$ & Psychology combined medicines & Hormone therapy & 6 months & (1)(2) \\
\hline Zhang et al 2008 & $25 / 24$ & Psychology combined medicines & Medicine & 3 months & (7) \\
\hline Wang et al 1998 & $50 / 50$ & Psychology combined medicines & Medicine & 1 months & (1)(2)(5) \\
\hline Wang et al 2005 & $32 / 31$ & Psychology combined medicines & Doxepin & 2 months & (1)(2) \\
\hline Zhuo et al 2010 & $120 / 60$ & Psychology combined medicines & Medicine & 6 months & (6)(7) \\
\hline Wang et al 2006 & $30 / 30$ & Psychology combined medicines & Medicine & 6 months & (7) \\
\hline Xu et al 2012 & $45 / 44$ & Psychology combined medicines & Hormone therapy & 6 months & (6) \\
\hline Yang et al 2012 & $34 / 34$ & Psychology combined medicines & Medicine & 2 months & (3)(4) \\
\hline Xu et al 2012 & $32 / 33$ & Psychology combined medicines & Medicine & 3 months & (3)(4) \\
\hline Yu et al 2013 & $20 / 20$ & Psychology combined medicines & Oryzanol & 1.5 months & (7) \\
\hline Ai et al 2013 & $40 / 40$ & Psychology combined medicines & Hormone therapy & 2 months & (5) \\
\hline
\end{tabular}

Notes: T: treatment group; C: control group; Result Index index: (1)SCL-90 anxiety factor; (2)SCL-90 depression factor; (3)SAS scores; (4)SDS scores; (5)Kupperman menopausal scores; (6)Serum hormone level: blood E2,FSH and LH levels; (7)Total effective rates. 
TABLE II META-ANALYSIS OF EFFECTIVENESS

\begin{tabular}{|c|c|c|c|c|c|}
\hline Result Index & Events & Heterogeneity analysis & $\begin{array}{l}\text { Meta-analysis } \\
\text { model }\end{array}$ & Mean difference & $\mathrm{P}$ \\
\hline SCL-90 anxiety & 12 & $\mathrm{P}=0.02 .12=99 \%$ & Random & $\begin{array}{c}\mathrm{MD}=0.27, \\
95 \% \mathrm{CI}(0.04,0.49)\end{array}$ & 0.02 \\
\hline SCL-90depression & 12 & $\mathrm{P}<0.0001, \mathrm{I} 2=92 \%$ & Random & $\begin{array}{c}\mathrm{MD}=0.28 \\
95 \% \mathrm{CI}(0.15,0.41)\end{array}$ & $<0.0001$ \\
\hline SAS & 5 & $\mathrm{P}<0.00001, \mathrm{I}=92 \%$ & Random & $\begin{array}{c}\mathrm{MD}=8.54, \\
95 \% \mathrm{CI}(6.21,10.86)\end{array}$ & $<0.00001$ \\
\hline SDS & 6 & $\mathrm{P}<0.00001, \mathrm{I}=99 \%$ & Random & $\begin{array}{c}\mathrm{MD}=8.54 \\
95 \% \mathrm{CI}(6.21,10.86)\end{array}$ & $<0.00001$ \\
\hline Kupperman scores & 3 & $\mathrm{P}<0.00001, \mathrm{I} 2=0 \%$ & Fixed & $\begin{array}{c}\mathrm{MD}=5.55 \\
95 \% \mathrm{CI}(4.76,6.34)\end{array}$ & $<0.00001$ \\
\hline$E^{2}$ & 2 & $\mathrm{P}<0.29, \mathrm{I} 2=70 \%$ & Random & $\begin{array}{c}\mathrm{MD}=9.18, \\
95 \% \mathrm{CI}(-7.96,26.32)\end{array}$ & 0.29 \\
\hline FSH & 2 & $\mathrm{P}=0.31, \mathrm{I} 2=98 \%$ & Random & $\begin{array}{c}\mathrm{MD}=8.69, \\
95 \% \mathrm{CI}(-7.99,25.37)\end{array}$ & 0.31 \\
\hline LH & 2 & $\mathrm{P}<0.0001, \mathrm{I} 2=0 \%$ & Fixed & $\begin{array}{c}\mathrm{MD}=2.31 \\
95 \% \mathrm{CI}(1.17,3.45)\end{array}$ & $<0.0001$ \\
\hline Total effective rate & 12 & $\mathrm{P}<0.00001, \mathrm{I} 2=0 \%$ & Fixed & $\begin{array}{c}\mathrm{OR}=7.32, \\
95 \% \mathrm{CI}(5.11,10.50)\end{array}$ & $<0.00001$ \\
\hline
\end{tabular}

\section{DISCUSSION}

\section{A. Effectiveness Analysis}

Twenty-eight articles were analysed in this research, showing psychological intervention can effectively improve perimenopausal symptoms, relieve negative emotions and improve clinical efficacy ${ }^{[2,5-31]}$. It was found psychological state and quality of life were significantly improved with psychological intervention in Zufang $\mathrm{Xu}(2012)$ assay. Meiqing $\mathrm{Xu}(2012)$ found blood levels of E2, FSH and LH in patients of psychological intervention group and control group improved compared with those before treatment, and the difference was statistically significant $(\mathrm{P}<0.01)$. However, no significant difference were found between after treatment $(P>0.05)^{[27]}$. In addition, researches found the control and improvement were better than those with drug treatment alone for patients who adopt hormone replacement therapy combined with psychotherapy and nursing ${ }^{[31]}$. It can be seen from this:

- Psychological intervention is effective in improving the psychological and quality of life of perimenopausal syndrome patients. And no substantial impacts on hormone changes with mental intervention, only used as an auxiliary treatment. However, drug therapy is still mainly relied on in treatments of perimenopausal syndrome with endocrine disorders.

- Hormone replacement therapy can only improve physical symptoms, but cannot relieve psychological distress of patients.

\section{B. Significance of Research}

Perimenopausal syndrome is a common gynecological disease among women. With the change of medical model, hormone replacement therapy can't completely eliminate its symptoms, which makes people begin to pay attention to the influence of social psychological factors on perimenopausal women. Patients with perimenopausal syndrome are treated with conventional drugs and targeted psychological intervention, with psychological support received and negative emotions vented. At the same time, patients are helped to establish a new positive cognitive mode, which provides theoretical knowledge support for them by changing their unhealthy cognitive mode. Results showed patients combined with psychological intervention can better eliminate panic and anxiety of perimenopausal syndrome, facing and accepting their own physiological and psychological changes, compared with simple drug therapy. Moreover, it enables them to enjoy their real life with more positive attitude and enhance their immunity to adverse emotions and events. Therefore, psychological intervention can improve the symptoms, psychology and curative effect of perimenopausal syndrome patients, which has positive clinical guiding significance and is worthy of promotion and application.

\section{Limitations of Evidence, Quality and Research}

Although 6 of the above studies are of Grade B quality, most of them are Grade C, so the overall study is still lowgrade evidence. However, as a non-drug therapy, no toxic or side effects and wide ranges of applications, patients can received appropriate psychological comfort, encourages from members , and decreasing waste of clinical resources. What's more, it accords the rules of "psysicalogy-mental-social medicine", with "medium recommendation".

There are still some limatations. First, the overall level of literature quality is low, and most studies have problems of unclear random methods and allocation, which may lead to selective bias, and difficult to achieve double blind, which may lead to implementation bias and measurement bias. Second, specific methods of psychological intervention were not classified in the included study. The clinical symptoms, emotional degree and duration of intervention before the intervention were not unified. So did different of the levels of interveners. These might be sources of heterogeneity. It would have more clinical significance if it can explored which intervention method is more suitable and make detailed classification and meta-analysis. It is expected that domestic research will pay more attention to the report of research methods and report more detailed high-quality randomized controlled trials, in order to increase the strength of evidence. 


\section{ACKNOWLEDGMENT}

This research was financially supported by National Innovation and Enterpriseship Training Program (201710412004) and Scientific Researches Program of JXUTCM (2016jzzdxk011). The authors thank researches for providing experimental assistance with method applications. The authors also thank the anonymous reviewers for helpful comments.

\section{REFERENCES}

[1] Jie Le. Obstetrics and Gynecology. Beijing: People's Medical Publishing House, 2004, pp.382.

[2] Le Yan \&Peng Zhao. Psychological intervention in patients with perimenopausal syndrome. Maternal and child health. 2009 (3), pp.94-95.

[3] Siddiqui N I, Rabman S, Mia A R,et al. Evaluation of hormone replacement therapy. Mymensing Med Journal, 2005,14(2),pp.212-218.

[4] Sally Green, Julian PT, Philip Alderson, et a1.Cochrane Handbook Version 5.0.2.The Cochrane Collaboration.2008

[5] Yuelong Mo. Analysis of the role of psychological intervention in the treatment of female climacteric syndrome. Maternal and child health. 2014,8(3),pp.186-187.

[6] Min Deng \&Jianxiong GU. Psychological therapy of perimenopausal syndrome. Chinese rehabilitation theory and practice.2004,10(7),pp.430431

[7] Haibo Meng, Min Wang \& Minhui Li. Evaluation of mental status and psychological intervention effects of menopausal nurses. Chinese maternal and child health care.2011,26,pp.5576-5577.

[8] Jinhong Zhang, Junxia Gao, Jinhuan Gu, et al. Evaluation of cognitive behavioral nursing in menopausal syndrome, Qilu nursing journal. 2006,12(5),pp.814-815.

[9] Suzhi Lin. Psychological guidance for menopausal syndrome, Journal of Psychiatrist,2012(222),pp.47-48

[10] Shuwen Shen. Discussions on psychological nursing of menopausal syndrome, Contemporary nurse.2002(7),pp.51-52.

[11] Fuping Zhang, Chunlan Zhang, \& Cuicui Bai. Effects of nursing intervention on quality of life in patients with perimenopausal syndrome. Chinese journal of aesthetic medicine.2012,21(10),pp.470-471

[12] Daqing Li. Efficacy of Relieving depression on improvement of perimenopausal syndrome. Chinese journal of practical gynecology.2002,18(12),pp.737-738.

[13] Shujie Li. Psychological nursing of menopausal syndrome. Modern Chinese medicine application.2009,3(6),pp.170-171

[14] Aiqun Cui. Clinical observation of emotional support for menopausal syndrome. Hubei journal of traditional Chinese medicine.2007,29(10),pp.48-49.

[15] Chen Liu \& Libo Song. Observation on the cognitive therapy in the treatment of climacteric syndrome. Chinese practical medicine.2012,7(34),pp.228-229.
[16] Lanhua Liu. The role of cognitive therapy in the treatment of female climacteric syndrome. Chinese journal of neuropsychiatric diseases.2003,29(5),pp.362-363.

[17] Zufang $\mathrm{Xu}$. The impact of psychological care on the quality of life in menopausal syndrome patients. Journal of psychologist.2012(216),pp.264-265.

[18] Jiazhen Tian. Analysis of psychological support for perimenopausal syndrome. Chinese journal of practical neurology. 2007,10(9),pp.97-98.

[19] Yueying Hu. Efficacy of psychological intervention in perimenopausal syndrome. Chinese journal of preventive medicine. 2007,41(5),pp.424425 .

[20] Yuhong Zou. Effect of psychological intervention on the psychologica behavior of perimenopausal women. Health Road. 2013,12(10),pp.25-26.

[21] Xiuxia Wang. Effect of psychological intervention on the efficacy of perimenopausal syndrome. Modern preventive medicine.2008,35(21),pp.4163-4164

[22] Peiqin Zhang, Xiuyun Yan,\& Suxiu Li. Therapeutic effect of psychological intervention in the treatment of perimenopausal syndrome. China minkang medicine,2008,20(23),pp.2800.

[23] Guixia Wang, Aihua Ma, Shuncheng Li,et al. Therapeutic effect of psychological intervention on perimenopausal symptom groups. Journa of health psychology,1998,6(3),pp.314-315.

[24] Yiyun Wang, Lan Liu,\& Qiuyan Wang. Psychotherapy for menopausal syndrome. Chinese medical journal.2005,17(04), pp.186-187.

[25] Qinghua Zhuo \& Yunhua Ma. Therapeutic effect of Lily Gengnian Granule and Liweiai combined psychological intervention on perimenopausal syndrome. Shandong Medicine.2010,50(37),pp.85-86.

[26] Bin Wang. Comparison of the efficacy of oryzanol combined with psychological intervention and estrogen replacement therapy in the treatment of perimenopausal syndrome. Medical journal of new knowledge.2006,16(5),pp.306-307.

[27] Meiqing Xu. Clinical observation of hormone replacement combined with psychological counseling for perimenopausal syndrome. Chinese journal of rural medicine.2012,19(15),pp.25-26.

[28] Genhua Yang. Effect of paroxetine combined with psychological intervention on psychological status and well-beings in women with perimenopausal syndrome. Strait Pharmaceutical.2012,24(1),pp.184-185.

[29] Fangling Xu. 32 cases of perimenopausal syndrome in women with smal dose of Dailixin. Clinical medicine. 2012,21(21),pp.72-73.

[30] Hongbo Yu. Clinical observation of psychological intervention combined with oryzanol in the treatment of climacteric syndrome. Heilongjiang medicine.2013,26(4),pp.681-682.

[31] Yaqin Ai, Ljiun Zheng,\& Quanlin Yang. Effect of psychological intervention combined with hormone replacement therapy on perimenopausal syndrome. Journal of Hebei north university.2013,29(6),pp.91-93.

[32] Chen YH. Clinical experience of Kuntai capsule for treating patients with perimenopausal symptoms. Zhong Yi Zhong Yao 2011; 29(18): 113-114. 